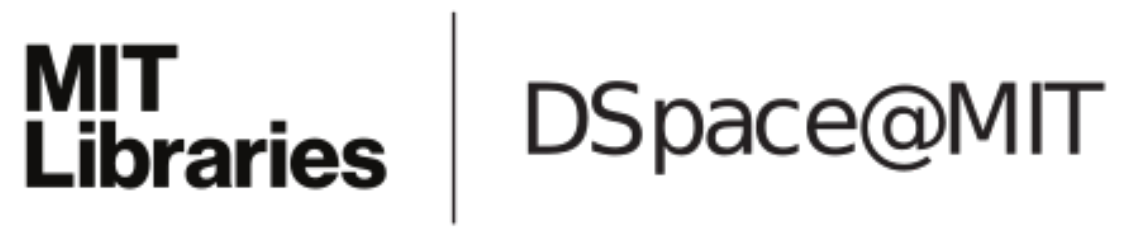

MIT Open Access Articles

Technical developments of functional electrical stimulation to correct drop foot: Sensing, actuation and control strategies

The MIT Faculty has made this article openly available. Please share how this access benefits you. Your story matters.

Citation: Melo, P.L. et al. "Technical Developments of Functional Electrical Stimulation to Correct Drop Foot: Sensing, Actuation and Control Strategies." Clinical Biomechanics 30, 2 (February 2015): 101-113 @ 2014 Elsevier Ltd

As Published: http://dx.doi.org/10.1016/J.CLINBIOMECH.2014.11.007

Publisher: Elsevier BV

Persistent URL: http://hdl.handle.net/1721.1/114594

Version: Author's final manuscript: final author's manuscript post peer review, without publisher's formatting or copy editing

Terms of use: Creative Commons Attribution-NonCommercial-NoDerivs License 


\title{
Technical Developments of Functional Electrical Stimulation to Correct Drop Foot: Sensing, Actuation and Control Strategies
}

\author{
P. L. Melo ${ }^{1,2}$, M. T. Silva ${ }^{1}$ J. M. Martins ${ }^{1}$ and D. J. Newman ${ }^{2}$
}

${ }^{1}$ IDMEC, Instituto Superior Técnico, Universidade de Lisboa, Av. Rovisco Pais, 1, Sala 1.02, 1049-001

Lisboa, Portugal. Corresponding author: paulo.de.melo@tecnico.ulisboa.pt, +351969212926.

${ }^{2}$ Man-Vehicle Lab, Massachusetts Institute of Technology, 77 Massachusetts Avenue, Cambridge, MA, United States of America 02139

\begin{abstract}
This work presents a review on the technological advancements over the last decades of functional electrical stimulation based neuroprostheses to correct drop foot. Functional electrical stimulation is a technique that has been put into practice for several years now, and has been shown to functionally restore and rehabilitate individuals with movement disorders, such as stroke, multiple sclerosis, traumatic brain injury, among others. The purpose of this technical review is to bring together information from a variety of sources and shed light on the field's most important challenges, to help identifying new research directions. The review covers the main causes of drop foot and its associated gait implications, along with several functional electrical stimulation-based neuroprostheses used to correct it, developed within academia and currently available in the market. These systems are thoroughly analyzed and discussed with particular emphasis on actuation, sensing and control of open- and closed-loop architectures. In the last part of this work, recommendations on future research directions are suggested.
\end{abstract}

Keywords: Functional Electrical Stimulation, drop foot, neuroprosthesis, gait, closed-loop control, open-loop control.

\section{Introduction}

Stroke is among the four leading causes of death and disability worldwide, with about 15 million people suffering stroke every year. Of these, one third dies and another third becomes permanently disabled [1]. Depending on the size and location of the lesion, stroke survivors can have their physical and/or mental capabilities impaired. Motor disabilities are often a consequence and can affect speech, grasp and gait, as well as other everyday functions. With a prevalence of about 20\% amidst stroke survivors, drop foot (DF) is one of such disabilities that severely impairs these persons' mobility [2]. Along with stroke, cerebral palsy (CP), multiple sclerosis (MS), traumatic brain injury (TBI) and spinal cord injury (SCI) are also neurological conditions that can lead to DF. This condition is often the result of a paralysis and/or weakness in the patient's dorsiflexor muscles, making him unable to clear the toes off the ground during the swing phase of gait. Due to this lack of proper muscle activation, compensatory mechanisms at other joints, such as the knee or the hip, are often present and result in a very typical steppage or hip hiking gait [3]. Slap foot is another condition that is often concurrent with DF. Characterized by an uncontrolled plantarflexion, right after initial contact (heel strike), slap foot can lead to chronic ulcers [4]. Additionally, muscle weakness and/or spasticity at the plantarflexors might also occur, resulting in an inability to support their own weight.

Often DF individuals still retain electrically excitable peripheral nerves and muscle tissues, which allows the use of techniques such as Functional Electrical Stimulation (FES) to restore their lost mobility. FES is a technique that taps into the person's paralyzed muscles to produce movements that would not be possible otherwise. Over the years, FES has proven itself as a promising technique to restore lost motor functions, allowing neuromuscular impaired individuals to recover lost motor functions, 


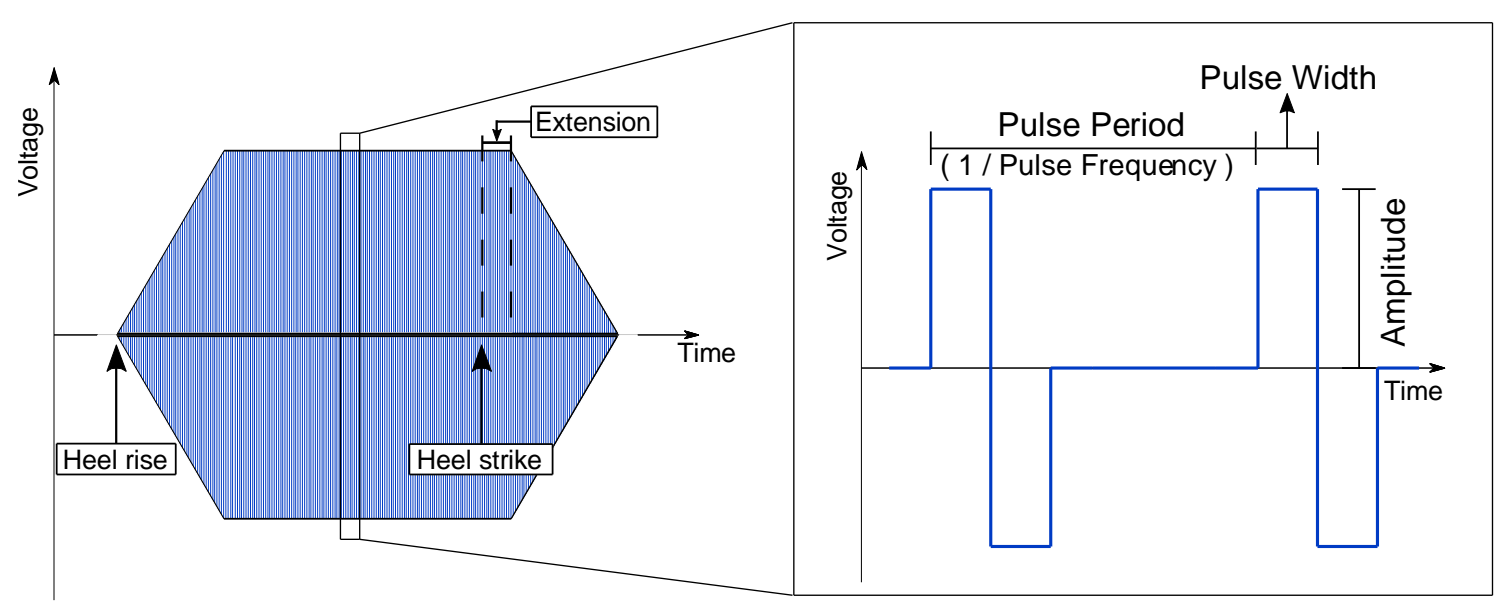

Figure 1 Typical trapezoidal waveform used by most FES commercial systems, with balanced charges, posing no threat to tissue integrity. Note: Figure not drawn to scale.

positively impacting their quality of life [5]. FES was first used to correct DF in the 1960s [6]. Since then, this research field kept growing and eventually the first FES-based DF neuroprostheses became commercially available [7-9]. However, and despite continuous developments, there are still important challenges to be tackled, specifically on the control architectures aspects of these types of neuroprostheses [10,11]. Essentially, a FESbased neuroprosthesis to properly correct DF, and its associated conditions, should at least provide foot clearance during the swing phase, minimize foot slap during controlled plantarflexion (loading response) and, if necessary, provide assistance to the plantarflexors during push-off.

This work presents a comprehensive review of the latest FES-based DF developments to help identifying new research directions, with emphasis on different actuation and sensing strategies, specifically focusing on open- and closed-loop (feedback) control architectures. Recommendations on future research directions are also discussed. A thorough review on earlier FES systems to correct DF since the 1960s up to 2001 can be found elsewhere [12]. Functional electrical stimulation as a rehabilitation tool has been reported to improve gait when combined with conventional therapies [13-17], however it is not going to be addressed as a main topic, since it falls outside the scope of this article. Nonetheless, therapeutic effects of FES may be brought to discussion when necessary. The combined use of FES, as a neuroprosthesis, with orthoses, often named hybrid orthoses, will again not be the main focus of this review, despite its increasingly and promising use in the last few years [18-20], specifically when FES alone is not enough to provide the desired function or support, which most often occurs in more complex conditions than DF, such as paraplegia.

\section{Using FES to correct Drop Foot}

The typical architecture for a FES-based DF neuroprosthesis can be seen as an integration of a network of sensors, a control algorithm and a stimulation unit. The sensing network should always provide system information to the controller [21]. This controller should then be able to correctly adjust its inputs to the stimulation unit. Thus, optimal control strategies to correct DF should be sufficiently robust to the nonlinear, time-varying and coupled response of stimulated muscles [10]. Furthermore, electrical stimulators should be portable, lightweight and flexible enough, in terms of specifications and parameters, to deal with different control strategies requirements. Tables 1 and 2, presented in appendix, show detailed information on portability, types and stimulation characteristics of several research and commercial stimulators, respectively.

To stimulate nerve fibers and generate more efficient muscle contractions, a rectangular shaped electrical pulse has been suggested as optimal, since it overcomes the problem of the nerve fiber membrane accommodation (see Figure 1, a typical stimulation pulse). Moreover, the pulse should provide an equal distribution of charges at the electrodes locations during stimulation period, so that no electrochemical imbalance occurs, eventually leading to body tissue damage [22]. This is usually achieved by having a pulse in one direction (positive phase) and another one in the opposite direction (negative phase), symmetric or not. Additionally, on a typical stimulation profile, there is often a ramping up and down of the stimulus, so that sudden responses are avoided 
and more physiological type of contractions are achieved [23]. To a certain degree, prolonging the stimulus for a small amount of time after heel strike, has been used to help controlling slap foot [24]. The frequency of the pulse controls the type of muscle contraction and the amount of force produced. Pulse amplitude and width, represent how much, and for how long, current is needed to produce a minimal amount of ionic flow to trigger action potentials. Further information on these parameters can be found elsewhere [22].

Currently, most existing ankle-foot orthoses are passive. The only active systems commercially available are FES-based. Mechanically-based active DF orthoses are still to surface outside the research setting [25]. Until now, all the commercially available FES systems have been solely based on open-loop architectures. Even though most of these systems use sensory feedback to switch between states (e.g. finite state machine controllers, FSM), they should not be considered closedloop controllers; instead, a closed-loop system should be defined as a system where the controller is sufficiently stable and robust to correct for model errors and external disturbances, such as an obstacle or muscle fatigue [11]. Tables 3 and 4 summarize several approaches using open- and closed-loop strategies, respectively.

\subsection{Open-loop systems}

The first open-loop system to correct DF using FES was developed by Liberson et al. [6]. Liberson's system enabled dorsiflexion of the foot by synchronous stimulation of the tibialis anterior muscle during the swing phase (see Figure 1). This type of system was a FSM, in this particular case with two states, stimulus on or off, which were detected by means of a shunt resistor to sense heel contact. To the present date, Liberson's concept has remained very popular among researchers and most of the systems built in the following decades were based on his FSM architecture.

\subsubsection{Research prototypes}

A. Constant preset stimulation control based on foot switches.

The advent of microcontrollers in the 1970 s made possible the continuous development of more flexible and smaller stimulation devices. One such example is the system developed by Malezic et al. [26], where microcontrollers were used to develop a four channel surface stimulator system. The system was evaluated as therapeutic and orthotic device in twenty-one subjects, 11 with stroke and 10 with TBI. The orthotic system relied in heel switches, to detect the swing and stance phases. Preset stimulation pulses with constant amplitude, width and frequency (see Figure 1) were delivered to the common peroneal nerve (tibialis anterior, TA, stimulation), producing dorsiflexion during the swing phase. Other stimulation channels were used to enable extra knee flexion/extension, hip extension and reciprocal arm swing. Malezic's work showed, for both patients' groups, gait improvements of $22 \%, 10 \%$ and $9 \%$ in velocity, cadence and stride length, respectively. Very recently, Chou et al. [27] performed similar experiments to Malezic's reciprocal arm swing trials, with triceps brachii stimulation.

In the line of Malezic's work, Kim et al. [28] combined the traditional ankle dorsiflexors stimulation with hip abductors stimulation, in a population of thirty-six patients with post stroke hemiparesis. Foot switches enabled TA stimulation during swing phase and gluteus medius stimulation during the stance phase, to prevent the pelvis from dropping to the opposite side. Kim reported improvements against only TA stimulation and no stimulation, of up to $48 \%$ in gait symmetry, $15 \%$ in velocity, $7 \%$ in cadence and $7 \%$ in stride length.

Sabut et al. [29] performed an eight-week randomized controlled trial (RCT) on ten DF patients, using a heel switch to trigger TA stimulation during the swing phase. Gait was statistically improved when the orthotic device was used, with increases in gait velocity, cadence and stride length of $11.7 \%, 10.8 \%$ and $13.3 \%$, respectively. There was also a reduction in the physiological cost index (PCI) of $17.7 \%$. Additionally, there were some therapeutic effects with a significant decrease of spasticity, increased range of motions, enhanced blood circulation and reduced pain.

\section{B. Modulated preset stimulation control based on foot switches.}

The open-loop systems presented until now have been surface based and with a stimulation profile consisting of a constant preset train of pulses. O'Halloran et al. [30,31] adopted a different stimulation strategy and developed a heel switch triggered implantable system for DF with pulse width modulation across two gait phases. Tested in one hemiplegic subject, the implantable stimulator had two independent channels, in a 12-polar nerve cuff electrode, to be placed on the peroneal nerve controlled 


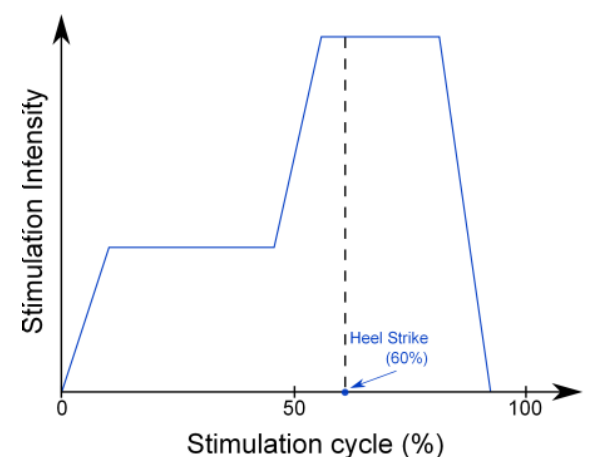

Figure 2 Modulated stimulation profile [30]. Adapted and reproduced with permission from Elsevier.

externally by induction. By proper balance between these channels, a more physiological dorsiflexion was achieved. With the proposed stimulus profile (see Figure 2), O'Halloran showed that increase of stimulation intensity just before the heel strike, enabled a better controlled plantarflexion during loading response, minimizing the possibility of foot slap. It was even reported that patients would possibly benefit, if stimulation was extended beyond this point. Similarly Breen et al. [32] developed an interesting DF stimulator system using two foot switches (heel and toe) and a modulated stimulus envelope profile, with stimulation timings being adjusted from cycle to cycle based on gait velocity.

\section{EMG-modulated preset stimulation control based on foot switches.}

The previously discussed works on modulation of the stimulus envelopes essentially based profile adjustments on clinician's specifications. Lyons et al. [33] through a "natural" stimulation strategy, used offline surface electromyography (sEMG) to modulate the profile shape for one hemiplegic DF patient. This approach resulted in significantly higher dorsiflexion range, going up to $7.68^{\circ}(76 \%)$ against $4.35^{\circ}(53 \%)$ of the traditional approach (see Figure 1). These improved results might have been the result of a less severe spastic reaction of the calf muscles during dorsiflexion. It is important to notice that the latter approach is less efficient than the "natural" one, since it requires the approximately double of the charge to produce a less efficient dorsiflexion. Adding on the previous work, O'Keeffe et al. [34] developed a similar strategy. Although his system was only tested in a healthy subject, it was also reported as more energetically efficient, by using $47 \%$ less stimulation current than traditional stimulation envelopes.
D. Preset stimulation control based on inertial sensors and force sensitive resistors.

Alternatives to foot switches, such as inertial sensors, have been surfacing over the last decades allowing new FES control strategies. Weber et al. [35] tested such a system, the Walkaide, a surface-based commercial stimulator with a tilt sensor. Weber also modified Walkaide to be able to integrate a new type of implantable microstimulator called BION (BIOnic Neuron). BIONs were developed by Loeb et al. [36,37] as a minimally invasive self-contained micro-stimulation platform for a large range of applications (see Figure 3), easily injected near target muscles or nerves. The latest generation includes built-in EMG, accelerometers and inter-device communication features and can last a few days with a single charge [36]. Together with a shank accelerometer and control algorithms within the Walkaide system, Weber showed that BIONs were a reasonable alternative to surface systems. Tested on a 42 year old incomplete SCI male, the prototype produced an almost normal dorsiflexion during the swing phase, greatly increasing gait speed and significantly decreasing PCI. The dorsiflexion was even more balanced than that of surface-based systems (where often the foot is inverted), since an additional BION was inserted at the peroneus longus muscle. Interestingly, the prototype fell behind the surface FES system in both gait speed and PCI.

In 2011, Miura et al. [38] developed a wireless surface FES rehabilitation system, fully based on inertial sensors. The system was composed of a wireless surface 1-channel stimulator and seven wireless inertial sensors (thigh, shank and foot in both legs plus the lumbar region). Tested successfully in one hemiplegic patient with DF, the system provided enough foot clearance similar to the levels of the non-paretic side, by only using the vertical axis

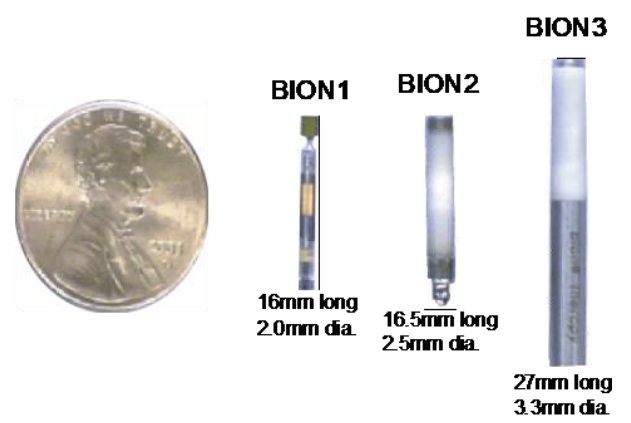

Figure 3 Three generations of implantable BION microstimulator [36,106]. Reproduced with permission from Rockwater, Inc. 


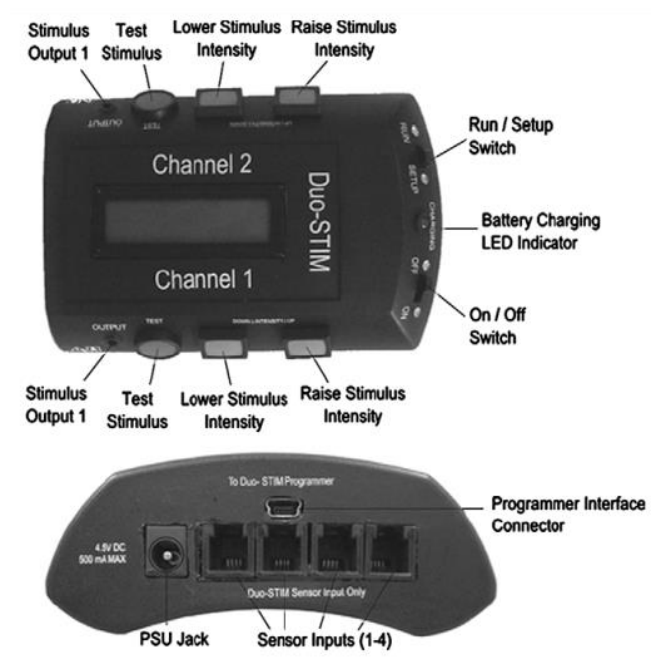

Figure 4 Duo-STIM system for drop foot [39]. Reproduced with permission from Elsevier.

of the shank accelerometer to trigger stimulation

Another system with inertial capabilities was developed by Breen et al. [39]. Called DuoSTIM, the system was a full-fledged portable dual channel stimulator with inertial sensors (see Figure 4). The device comes with embedded control algorithms to correct DF based on foot switches (heel and toe), but allows any free-form stimulation envelope and additional control strategies based in different sensors, such as accelerometer-based stimulation. The device has been cleared for clinical trials, but at the time this article was written no trials had been performed.

Moving away from traditional DF correction and making use of inertial sensors, Dosen et al. [40] developed a control strategy based on decision trees. Machine learning techniques were used to estimate several states, by using three inertial sensors (shin, shank and foot) and force measurements (heel and toe) as input data and sEMG of four muscles as output data (stimulation on or off). Although the system was only bench tested, the authors reported that the control based on decision trees, has the advantage of being human readable and interpretable, allowing better customization by the clinician.

\section{E. Constant preset stimulation control based on natural sensors.}

Over the last two decades, the use of whole nerve cuffs to record the response of cutaneous mechanoreceptors (natural sensors) has been proposed as a way of detecting main gait events, such as heel strike. This "natural foot switch" was first implemented by Haugland et al. [41] and it was composed by a $30 \mathrm{~mm}$ cuff implant placed at the sural nerve. Tested in a hemiplegic patient with DF using a surface stimulation system, the device proved its feasibility, despite some reliability issues with heel strike detection, which were dealt by implementing a timer to match the stance phase. Continuing his work, Haugland developed an implantable nerve stimulator, small enough to fit into the wall of a nerve cuff electrode. Results showed the device good selectivity for the peroneal and tibial fascicles [42].

Later on, Hansen and Haugland [43] picked up on Haugland's work and integrated the implantable stimulator with sural nerve recordings to correct DF. In his concept (see Figure 5), Hansen improved the earlier low detection rates by using neural activity and force sensitive resistors (FSR) data to train machine learning algorithms, specifically adaptive logic

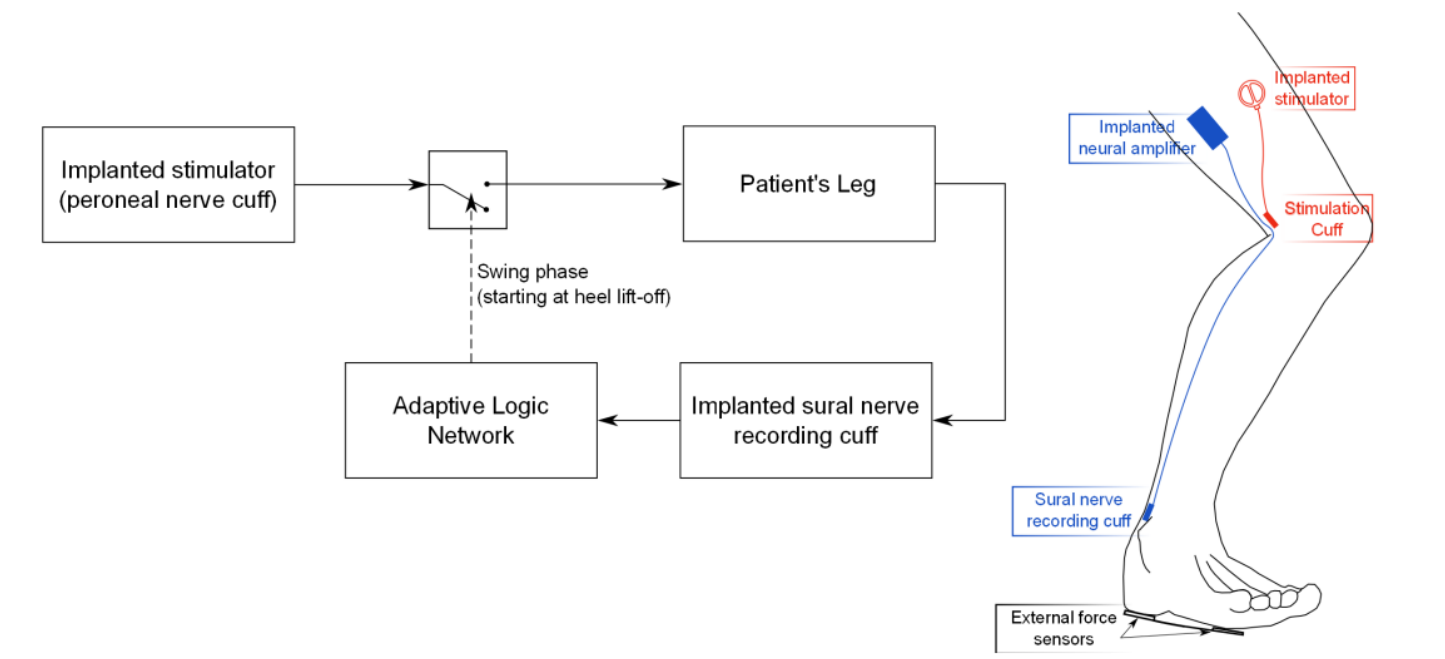

Figure 5 Implantable system for DF correction using Adaptive Logic Networks [43]. 
networks (ALN). Gait trials were performed on a 32 year old female with drop foot using the traditional trapezoidal stimulation, with the ALN having an acceptable functional performance as well as stability over a period of several months.

In 2005, Hoffer et al. [44] performed one of the first clinical trials with a fully implantable device to correct DF, the Neurostep system (Neurostream Technologies). However, at the present time, Neurostep is still an investigational medical device under clinical trials. The Neurostep system works autonomously without any external device, with the batteries, sensors and stimulation units all included in an implantable package. Stimulation occurs at the common peroneal nerve, though gait events are detected using tibial nerve recordings. A 70 years old male with hemiplegic DF that could only walk for 5 to $10 \mathrm{~m}$ without fatigue, had the device implanted and after 6 months his walking range increased to $250 \mathrm{~m}$. Despite this achievement and the initially high detection rates that ranged from 72 to $90 \%$, the sensing cuffs' impedances degraded over time, which led to increasingly lower detection rates.

\subsubsection{Commercial systems}

Currently, the only active ankle-foot orthoses to correct drop foot available in the market are FES-based. These systems range from externally worn portable surface stimulators, to partially implantable solutions, where only the stimulator/electrodes are implanted. There is no fully implantable system yet. All these systems are open-loop, since they only use sensors to time the stimulation (based on a FSM type of control), which in all cases is a preset stimulus pulse train tuned by a qualified clinician.

There are currently three well-known surface FES-based solutions, the Odstock, the Ness L300 and the Walkaide; and only two partially implantable solutions, the STIMUSTEP and the ActiGait systems. Interestingly, one of the first FES commercial systems (no longer is) to correct DF was partially implantable (see Figure 6). Developed in 1971 by Medtronic, Inc. (Minneapolis, Minnesota), the device was tested in 16 patients over a 3 years period, which were able to achieve ankle neutral position (some with inversion) during the swing phase and increase their gait speed by $37 \%$ compared to pre-surgery AFO speed [8]. Another similar commercial DF single channel surface stimulator that ceased production was the system developed by FES Medical Electronics (Glasgow, UK). An eleven week study performed with seventeen chronic

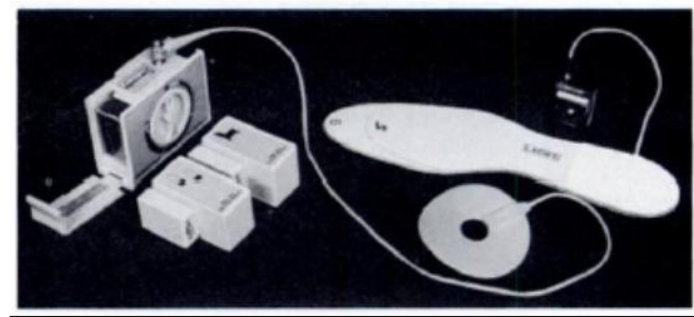

Figure 6 Neuromuscular Assist, the implantable system for DF correction developed by Medtronic, Inc. [8]. Reproduced with permission from Rockwater, Inc.

DF patients over a variety of different terrains showed significant orthotic improvements [45].

\section{A. Odstock Stimulators}

Odstock Medical (Wiltshire, UK) has had its presence in the market of surface FES-based solutions for 17 years. Currently, Odstock provides a variety of different stimulators, of which the Odstock Dropped Foot Stimulator (ODFS) and the Odstock Two Channel Stimulator (O2CHS) are the systems used to correct several gait conditions. The ODFS series is designed to correct DF, making use of a heel switch connected to a portable waist control unit. The O2CHS presents itself as a much more flexible device to tackle a variety of different neurological conditions, such as bilateral DF among others [24]. One hundred and fifty one patients with DF using the ODFS system, had their gait velocity increased by $27 \%$ and PCI reduced by $31 \%$ over a period of 4 and a half months [46,47]. Another study with 291 patients reported a generalized satisfaction with the device, reduced effort in walking over time with a decrease of $30 \%$ in PCI [48]. Significant carryover effects were reported in both studies for patients with non-degenerative disorders.

\section{B. Ness L300 System}

Commercialized by Bioness Inc. (Valencia, California, USA), the Ness L300 surface FES system includes the stimulation unit embedded in a below-the-knee cuff, a wireless heel switch and hand-held control for intensity control. With this device, Van Swigchem et al. [49] reported similar increases in gait velocity and cadence of 26 stroke patients compared to an AFO. FES was also reported as more comfortable, aesthetic and less fatiguing. 


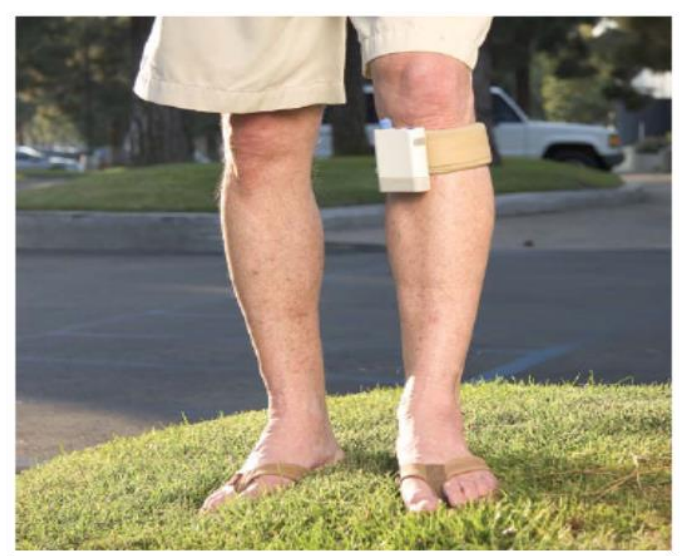

Figure 7 The Walkaide system to correct DF [23]. Reproduced with permission from the Journal of Automatic Control.

\section{Walkaide System}

The Walkaide is also a below-the-knee cuff (see Figure 7), however it uses a vertical axis accelerometer-based gait detection algorithm to trigger the stimulation based on acceleration thresholds. This small and self-contained singlechannel stimulation system is capable of autonomously tuning itself for each subject [50]. Experiments performed with the device in 73 patients, reported a sufficient DF correction, with up to $15 \%$ increases in gait velocity. The device's simplicity was a major advantage pointed out by most of the patients [23].

\section{STIMuSTEP System}

The STIMuSTEP system (Finetech Medical Ltd., UK) is a passive implantable dual channel peroneal nerve stimulator triggered by a foot switch. The extra stimulation channel balances dorsiflexion by everting the foot. In a RCT conducted by Kottink et al. [51] with 29 DF patients over 26 weeks, increases of $23 \%$ in gait velocities were reported, compared to a $3 \%$ increase in the control group. Additionally, patients also reported a more comfortable gait.

\section{E. Actigait System}

The other partially implantable solution commercially available is the Actigait system, developed by Neurodan A/S (Aalborg, Denmark), a subsidiary of Ottobock group (Berlin, Germany). The Actigait system uses the same control principles as STIMUSTEP, however the system's main parameters differ (see Table 2), as well as the heel switch, which is wireless. In a study with 15 stroke survivors over 15 months, Burridge et al. [52] reported significant increases in mean gait distance and velocity, despite occasional reliability issues with radio-frequency communication of the heel switch. Further studies performed by Van Swigchem et al. [53] in one stroke survivor showed that Actigait had similar performance compared to Ness L300 and an AFO, but an almost symmetric gait.

\subsection{Closed-loop systems}

As early as 1974, closed-loop strategies have been proposed to correct DF. However, several technological problems at the time, made their clinical applicability difficult [54]. Despite most developed systems until now have successfully implemented open-loop architectures, they still do not provide performance levels of healthy individuals [55]. This is probably one of the reasons why other control strategies, such as closed-loop FES, have been pursued over the years [11]. Despite this increasing effort in the last decades to make closed-loop FES a reality in the clinical setting and outside academia, it is still a challenging task to control paralyzed limbs with FES, due to the coupled, nonlinear behavior and fatigue of electrically stimulated muscles.

\subsubsection{Trajectory tracking control}

Most closed-loop control strategies to correct DF developed to date have focused in making the foot follow a specific angle reference during gait. Kobravi and Erfanian [56] proposed such a solution by adopting a decentralized controller with an agonist-antagonist muscle pair (TA and calf muscles), controlled by pulse width modulation (see Figure 8a). Their strategy was based on two independent controllers for each muscle-joint dynamics, with the inter-subsystem interactions regarded as external disturbances. Each subsystem was based on an adaptive robust controller (ARC), composed by a fuzzy logic based sliding mode controller (SMC) and an adaptive linear compensator, to deal with system's nonlinear dynamics and chattering phenomena (see Figure 8b). In silico trials showed an excellent performance tracking the free ankle angle with an RMS error of $0.79^{\circ}$, a same order robustness against external disturbances $(1.1$ N.m constant torque at the joint) and against asymptotic muscle fatigue $\left(1.66^{\circ}\right.$ RMS error). Experiments were also performed in three neurologically intact subjects and three spinal cord injury subjects, with an excellent tracking achieved in all subjects, up to $3.40^{\circ}$ RMS errors. Again, the ARC was robust enough to cope with muscle fatigue and the presence of external disturbances. 

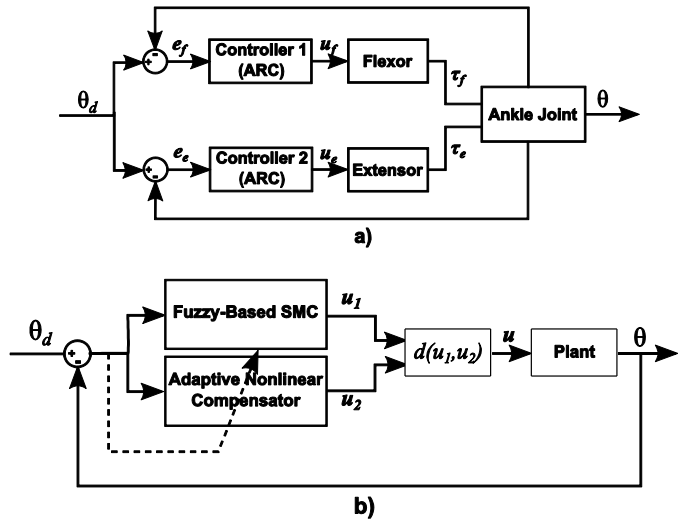

Figure 9 a) Decentralized control strategy for the ankle joint; b) Adaptive robust control, composed by an adaptive nonlinear compensator and a sliding mode controller (Kobravi and Erfanian 2009). Adapted and reproduced with permission from IOPscience.

Continuing Kobravi's work, Nekoukar and Erfanian [57] developed an adaptive terminal sliding mode closed-loop controller to track the ankle angle of two paraplegic subjects. Compared to Kobravi's work, this new controller had superior tracking performance in the same experiments (up to $1.3^{\circ} \mathrm{RMS}$ error), however it was tested in less patients and not as many trials were performed.

Nahrstaedt et al. [58] took a different approach on correcting DF and implemented a form of adaptive feedforward control, specifically a cycle-to-cycle iterative learning controller to track a preset ankle angle during the swing phase. At the beginning of each gait cycle, the pulse width modulated profile was updated, based on the angle error signal (feedback) from the previous cycle. Interestingly, to estimate ankle kinematics, Nahrstaedt developed a new type of sensor based in bioimpedance, which provided an almost linear tissue voltage drop as joint angle changes (see Figure 9). This technique was first employed as a joint angle sensor by Song et al. [59], and required hardware signal blanking during stimulation periods. Gait trials performed in one healthy subject did not produce a reasonable tracking, with convergence to an acceptable value occurring only after three cycles. This might have been the result of voluntary contractions of the healthy subject during stimulation.

Another closed-loop strategy was developed by Chang et al. [60] with the purpose of controlling the ankle trajectory during the swing phase, using an artificial neural network (ANN). To train the ANN, knee/ankle angles and stimulation amplitudes were used. Experiments were performed with success in healthy subjects, coupling the ANN with fuzzy logic under a foot switch based FSM controller and showed good

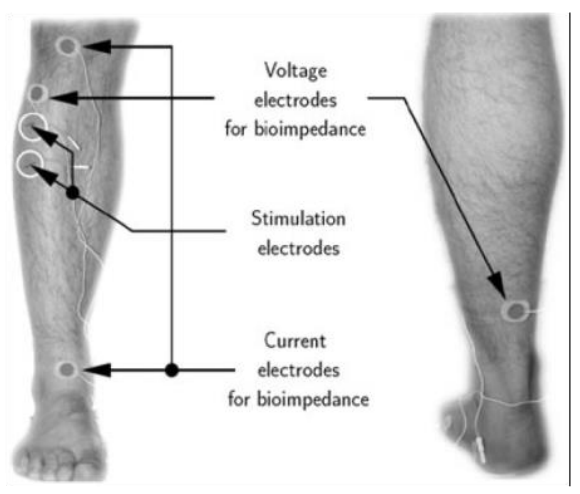

Figure 8 Electrodes' locations for the bioimpedance sensor and the stimulator [58]. Reproduced with permission from John Wiley and Sons.

tracking and convergence rate of the controller, when compared against a PID controller. Following the same line of research, Y. L. Chen et al. [61-63] also developed a closed-loop system to correct DF using a mixed ANN-fuzzy controller. Compared to the previously discussed system, this one does not use a foot switch based FSM. Instead, an ankle angle gait reference from a healthy subject is used. One other change included the implementation of the ANN in a feedforward manner, to provide a coarser estimate of the stimulus current based on a reference angle, with the feedback fuzzy controller's performing a fine tune of this current (see Figure 10). Once again, the fuzzy-ANN combination performed superior tracking $\left(3^{\circ}\right.$ to $4^{\circ} \mathrm{RMS}$ error) in three hemiplegic subjects when compared to only ANN and ANN plus PID.

Recently, Melo et al. [64] also used system identification techniques to identify intensityangle models that captured the linear dynamics of the electrically stimulated muscles, to perform closed-loop control of the foot in hemiplegic subjects. Using a custom built four-channel modular stimulator (see Figure 11) and an inertial sensing platform, the leg muscles were stimulated with a variety of different inputs: step, pseudo-random and random sequences. Preliminary results in healthy subjects using

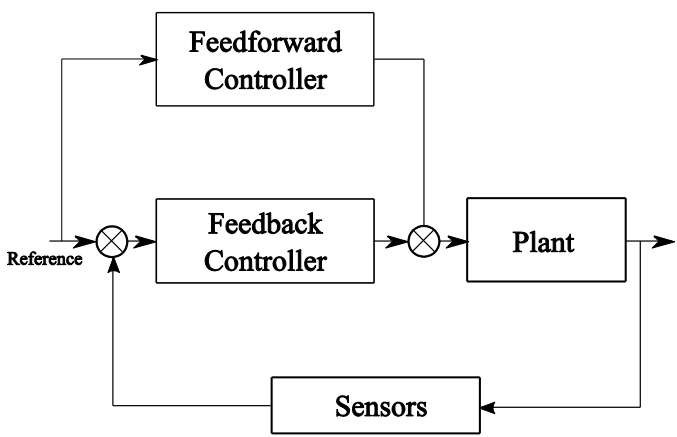

Figure 10 Generic control architecture based on feedback and feedforward controllers. 


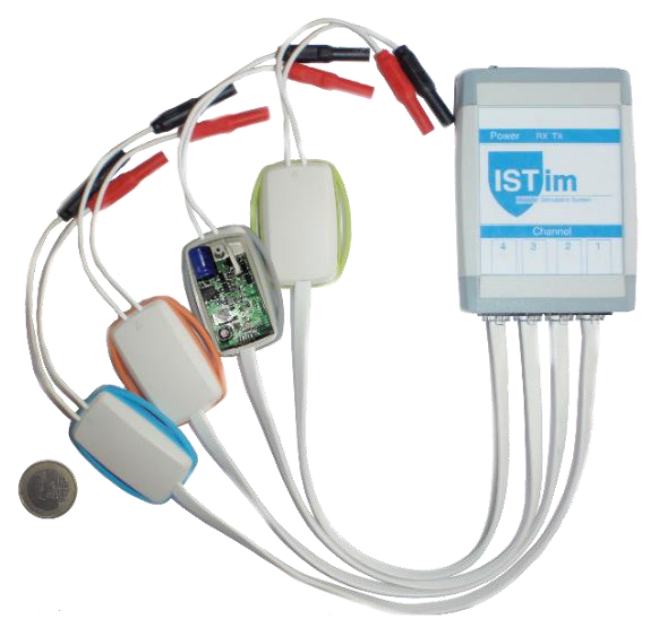

Figure 12 Multi-channel portable stimulator [64]. Reproduced with permission from the authors.

linear autoregressive exogenous models showed very accurate angle tracking with up to $90 \%$ (normalized RMS) in step response trials, and up to $63 \%$ for the random sequences. It is expected that nonlinear models will enhance the results. However, the current identified models are also expected to produce significant improvements in a closed-loop controller to correct DF.

Earlier, Y. L. Chen et al. [65] had developed another closed-loop solution to support gait of hemiplegic patients using a hierarchical feedback control architecture. In this control scheme (see Figure 12), an outer loop (controller $\mathrm{G}_{1}$ ) controls the trigger timings and amplitudes with foot switches and positions sensors. The inner loop (controller $\mathrm{G}_{2}$ ) performs FES stimulus parameters adjustments, based on a current sensor. A hemiplegic subject had his gait metrics improved significantly using this system, with the mean velocity, cadence and stride length increasing about 29\%, 60\% and $50 \%$, respectively. Continuing his work, Chen et al. [66] added a solenoid-based knee locker in the paretic leg that would lock during the stance phase. Reported results in one hemiplegic showed similar increases in gait velocity, cadence and stride length of $34 \%, 62 \%$ and $49 \%$, respectively.

Mourselas and Granat [67] compared an open-loop FSM system against a closed-loop FSM system. The FSM in both systems was based on FSRs and the low level controller of the closed-loop system used fuzzy logic to adjust the stimulus amplitude and match a desired target ankle angle. The trials showed that the closedloop controller had a superior performance, with higher number of steps with toe clearance and reduced step to step variability. Benedict and Ruiz [68] proposed a different control approach that was based on mirror control, meaning that unaffected limb signals were used as a desired

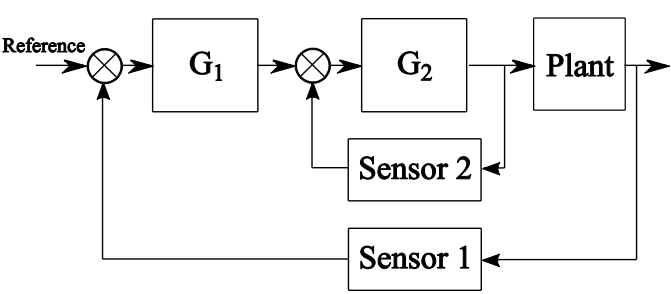

Figure 11 Generic hierarchical control strategy comprising an outer (controller $\mathrm{G}_{1}$ ) and an inner (controller $\mathrm{G}_{2}$ ) feedback loop.

target goals for the paretic limb. A bend sensor based FSM together with a PID controller were used to adjust the stimulus' amplitude. Although the system was not tested in hemiplegic patients bench tests were successful.

\subsubsection{Proportional sEMG control}

The control of FES-based orthoses to correct DF using sEMG has not been as extensively studied as the previously discussed systems, nevertheless there have been interesting developments within this topic. The purpose of such sEMG-driven control architectures is to provide a more physiological and continuous control the hemiplegic's ankle joint. This control strategy is based on the premise that patients still retain voluntary residual sEMG signals, which can be sensed by the system and used as inputs to the controller. The controller then uses the signal to proportionally stimulate the patient's muscles (see Figure 13). Simultaneous stimulation of a muscle and acquisition of its sEMG rises a new type of problem, since the stimulation produces an artifact in the readings of the sEMG, masking the actual muscle response signal. Yeom and Chang [69] described a new real-time stimulus artifact cancellation process using hardware blanking to remove the primary stimulation artifact and an adaptive Gram-Schmidt digital filter to remove the secondary artifact. The concept was successfully tested in one healthy subject. Similarly, Muraoka [70] also developed a patient-driven loop sEMG system, but again its efficacy was only proven with one healthy subject. W. L. Chen et al. [71] extended the previous concepts to the plantarflexors together with the dorsiflexors and tested in one subject with DF, which showed significant improvements in mean velocity, cadence, stride length of about $48 \%, 65 \%$ and $44 \%$ respectively. 


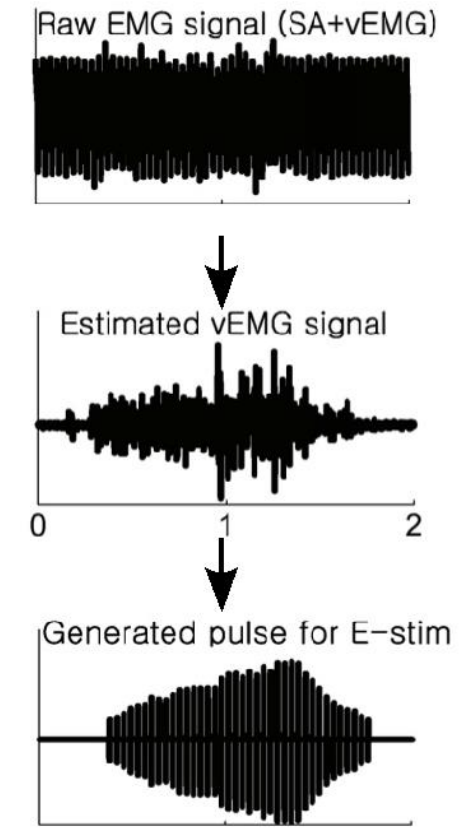

Figure 13 Example of the stimulus artifact (SA) present in EMG recordings from Yeom et al. The stimulation pulse profile extraction is based on the clean sEMG signal. Note: vEMG - voluntary electromyography; E-Stim - Electrical Stimulation. Adapted from Yeom and Chang [69], reproduced with permission from Elsevier.

\section{Discussion and conclusion}

Since 1961, when the first DF neuroprosthesis was developed by Liberson and his colleagues, FES has continuously been used as a tool to correct DF and its associated implications. Over the last decades, FES-based systems have had continuous developments, even though Liberson's concept remained very popular, with a large part of currently available commercial systems being based on his original 1961 architecture [72,73].

Advances in stimulation and sensing technologies as well as control strategies have made more efficient and reliable FES devices a reality. Compared to earlier DF FES-based systems, muscle stimulators evolved into more portable, and lightweight solutions, with current commercial system weighting less than $100 \mathrm{~g}$ $[23,73]$. Portable stimulators featuring multichannels are also becoming increasingly more common, allowing a higher number of muscles to be controlled during gait $[27,28,56,64]$. However, the well-established commercial surface-based FES solutions still rely only on single-channel stimulation of the TA during the swing phase $[46,50,73]$. It is interesting to note that some implantable solutions, such as STIMuSTEP [51] or Actigait [52], have similarly utilized one stimulation channel to produce dorsiflexion, but introduced additional channels to correct foot inversion, often concurrent with TA stimulation. In surfacebased systems this issue has also been addressed $[26,74]$.

A large part of DF FES-based systems developed to date have had their main focus placed on the correct timing of the stimulation onset during the swing phase (see tables 1 and 2 ). The use of FSRs to trigger stimulation has been one of the most popular approaches in the field of FES to correct DF [6,26,28,30,32]. Despite their ease of use and success detecting gait phases, foot switches often present reliability issues due to repetitive contact forces, which eventually lead to material degradation. On the other hand, natural sensors do not present these issues and have shown promising gait phase detection rates, but still not as high as it would be desirable $[41,42]$. To enhance these rates machine learning algorithms have been implemented [43]. Fully implantable solutions using natural sensors are still scarce, nevertheless there is currently one device under clinical trials [44]. In the meantime, partially implantable systems combined with external foot switches have proven their value, with currently two systems being commercialized.

Over the years, the development and implementation of more complex control algorithms resulted in a need to assess additional system states, other than the ones provided by the traditional foot switches. Examples that came up range from inertial [39,57,64], to position [56,60,67], sEMG [69-71], bioimpedance [58] and bend sensors [68]. Most of these sensing solutions have successfully been used to estimate desired physiological and/or kinematic states.

The control strategies of DF FES-based devices presented throughout this review, specifically open-loop architectures, have had their efficacy proven by existing commercial products. However, looking at normal healthy elderly men gait velocities of $1.2 \mathrm{~m} / \mathrm{s}$ with a stride of $1.3 \mathrm{~m}$ and stance phase of $63 \%$ [55], it seems open-loop FES-based devices can still be improved. In a large scale study, Kim et al. [28] reported velocities of up to $0.305 \mathrm{~m} / \mathrm{s}$ and stride lengths of $0.552 \mathrm{~m}$ and stance of $42.9 \%$. Similar results have been obtained in other studies $[23,29,46]$ Even though these were different studies and comparison between them against the mentioned standards is difficult to perform, it can be argued that there is still a significant difference between these metrics. Nevertheless, the combined developments in stimulation and sensing technologies over the last decades have been turning the possibility of closed-loop control strategies to improve those metrics a 
reality. In these two types of architectures, FSMs are often chosen as the high level controller, as summarized in tables 3 and 4. FSMs are often concurrent with foot switches, and in the case of closed-loop controllers, additional sensors usually provide inputs to a lower level controller.

Looking at open-loop architectures to correct $\mathrm{DF}$, most strategies, whether they are based on constant or modulated stimulus profiles, have in general achieved significant gait improvements $[26,28,34,35]$ as well as physiological cost indexes [29] when compared to unaided. These strategies have even improved other aforementioned gait complications associated with DF, such as foot slap [30] or the lack of support during the stance phase [28]. Several authors reported superior performance in systems where the stimulus profile was modulated when compared to the traditional trapezoidal profile. O'Halloran [30,31] showed that modulating the intensity during the swing phase and controlled plantarflexion in an openloop way, would avoid foot drop but would also minimize foot slap occurrences. Lyons et al. [33] and O'Keeffe et al. [34] also reported significant improvements against the trapezoidal envelope when using an EMG-modulated preset stimulation pattern, with the advantage of using around $50 \%$ less charge. It is important to note that the currently available solutions in the market are all open-loop FSMs [23,46,53]. Despite the significant gait improvements provided by current open-loop systems, there are still issues related with the nonlinear and timevarying behavior of the stimulated muscle, as well as fatigue, reflexes and spasticity [10]. In fact, several authors have reported closed-loop architectures to control ankle motion that are able to accurately track a desired reference and deal with muscle fatigue and external disturbances [57,60-62]. Although the number of patients tested was very low, another type of closed-loop control that has showed promising results is proportional sEMG control [69-71].

In general, whether it is surface or implantable-based, FES currently presents itself as one of the most promising techniques to recover lost motor functions resulting from upper motor neuron lesions. Implantable systems will generally provide better muscle selectivity [35], use smaller amounts of electrical charge and provide a more comfortable experience in terms of stimulus sensation, aesthetics and easiness of setup $[51,75]$. However, compared to a surface-based system, implantable solutions usually require surgery and there is always the risk of infection/rejection, along with issues regarding easiness of access in case of equipment malfunction.

Comparing the conventional passive AFOs to FES-based orthosis, there have been no clear and conclusive evidences that FES truly outperforms AFOs [76,77]. However, often patients show a greater degree of satisfaction with FES, since it is more comfortable, more aesthetic, less fatiguing and provide a better ankle balancing [49,78]. More benefits include the FES carry-over effect $[8,23,46]$.

Recommendations for future research on FESbased DF neuroprostheses

Although FES-based DF neuroprostheses have had continuous developments over the last five decades, there is still space for improvements since most of the currently available commercial open-loop solutions do not yet match the performances of healthy individuals, nor give support to other gait phases and muscles. As the next generation of FES neuroprostheses unfolds, we believe the focus should be on adaptive controllers that make use of model-based closed-loop architectures. This will allow robust systems to surface and be able to deal with the nonlinear and time varying dynamics of electrically stimulated muscles, as well as with external disturbances, muscle fatigue and spasticity.

Researchers are encouraged to keep developing multi-channel stimulators, to be able to control a higher number of muscles and better address the needs of closed-loop FES in DF correction. Systems with at least two channels should be considered when stimulating only the TA. These extra channels can be used to better balance the eversion/inversion of the foot, which sometimes can be difficult with only one channel [45]. This technique is often used in implantable systems, but not on surface ones. When manipulating FES stimulation parameters for control purposes, varying pulse width is recommended. Despite not being as physiological as frequency modulation, it produces more force and fewer fatigue with less stimuli [79]. Even amplitude modulation is preferred, since it uses less charge per second for a given force level [80]. When developing stimulators, it is also important to have sufficient range and spatial resolution in the FES parameters, so that closed-loop controllers can be effective. Regarding pulse width and frequency, ranges of $250 \mu \mathrm{s}$ and $60 \mathrm{~Hz}$ with unitary step resolution should be sufficient for most applications. It might be interesting to consider higher frequency ranges, as recent evidences have suggested that variable 
frequency stimulation trains up to $200 \mathrm{~Hz}$ are likely to enhance muscle performance [81]. In surface systems, biphasic pulses up to $120 \mathrm{~mA}$ (over a $1 \mathrm{k} \Omega$ load) of amplitude should be used, with at least unitary step resolution. However, implanted systems should have a resolution of one tenth of a milliampere or more, since a reasonable range is up to $2 \mathrm{~mA}$.

Concerning sensor options, the use of inertial measurement units, instead of FSRs, should be adopted whenever possible, since they provide a way to measure kinematics, which is often required for more robust control strategies. Additionally, inertial sensors have also been proved to be a practical solution as continuous gait phase detection systems [82].

Until now, closed-loop FES to correct DF has only been implemented using trajectory tracking or proportional sEMG strategies, however the central nervous system uses mechanical impedance modulation of the neuromuscular apparatus as a way to adapt to environmental conditions [83]. The development of adaptive impedance controllers using FES is therefore recommended. Such controllers have successfully corrected DF using active AFOs [25,84]. The use of neuromuscular models, such as Hill-type [85], in ankle foot prosthesis has been shown to nearly restore a natural gait in amputees [86]. Hence, porting these types of strategies to FES-based neuroprostheses might bring further improvements. In the case of model-based empirical strategies, progress in the recent years has been showing accurate control of knee and elbow joints using in nonlinear identification techniques, with either nonlinear autoregressive exogenous model $[87,88]$ and Hammerstein structures $[89,90]$. Although these models have proven to capture the dynamics of several muscle systems, these techniques have not yet been applied to the tibialis anterior muscle.

Despite open-loop FES has been the subject of several large scale studies, most of the closedloop systems implemented until now have been pilot experiments. Therefore, it will be important in the next years to increase these numbers, preferably with RCTs, so that the clinical application of these strategies becomes widely accepted. Along with this fact, it would be interesting to see the development of a universal performance framework for FES-based neuroprostheses, since it is often difficult to compare the efficacy of different studies, due to a lack of performance standards.

In the near future, when considering FES as the tool to rehabilitate a patient, we recommend that a surface-based system should always be the primary choice. Despite the fact that often these individuals have their sensory perception greatly diminished, an implantable solution should only be considered if the surface system produces unpleasant sensations, skin irritations, or if the patient is going to become a long-term user of the DF neuroprosthesis.

\section{Acknowledgments}

The authors would like to acknowledge IDMEC's researcher Daniel Simões Lopes for his fruitful discussions and also acknowledge Associação Salvador for their support, the support given by MIT Portugal Program and Fundação para a Ciência e Tecnologia through the DACHOR project - Multibody Dynamics and Control of Hybrid Active Orthoses (MITPt/BS-HHMS/0042/2008) and the research grant SFRH/BD/42966/2008.

\section{References}

[1] WHO. World Health Organization, The Atlas of Heart Disease and Stroke, Part Three: Global Burden of Stroke. 2004.

[2] Johnson CA, Burridge JH, Strike PW, Wood DE Swain ID. The effect of combined use of botulinum toxin type A and functional electric stimulation in the treatment of spastic drop foot after stroke: a preliminary investigation. Arch Phys Med Rehabil 2004;85:902-9.

[3] Don R, Serrao M, Vinci P, Ranavolo A, Cacchio A, Ioppolo F, et al. Foot drop and plantar flexion failure determine different gait strategies in Charcot-Marie-Tooth patients. Clin Biomech (Bristol, Avon) 2007;22:905-16.

[4] Hanft JR, Hall DT, Kapila A. A Guide To Preventative Offloading Of Diabetic Foot Ulcers. PodiatryToday 2011;24:60-7.

[5] Sheffler LR, Taylor PN, Gunzler DD, Buurke JH, Ijzerman MJ, Chae J. Randomized controlled trial of surface peroneal nerve stimulation for motor relearning in lower limb hemiparesis. Arch Phys Med Rehabil 2013;94:1007-14.

[6] Liberson WT, Holmquest HJ, Scott HJ, Dow M. Functional electrotherapy stimulation of the common peroneal nerve synchronised with the swing phase of gait of hemiplegic subjects. Arch Phys Med Rehabil 1961;42:101-5.

[7] Acimović-Janezic R, Gros N, Maležič M, Strojnik P, Kljajic M, Stanić U, et al. A comparative study of the functionality of the second generation of peroneal stimulators. Proc. 10th RESNA Conf., 1987, p. 621-3. 

Electrical Correction of Footdrop by Nerve of the Peroneal. J Bone Jt Surg 1975;57-A. odstock dropped foot stimulator: users and partner's perspectives. 7th Ann Conf Int ... 2002:1-2.

[10] Lynch C, Popovic M. Closed-loop control for FES: Past work and future directions. 10th Annu. Conf. Int. FES Soc., 2005, p. 2-4.

[11] Lynch C, Popovic. Functional Electrical Stimulation. IEEE Control Syst Mag 2008;28:40 50 .

[12] Lyons GM, Sinkjaer T, Burridge JH, Wilcox DJ. A review of portable FES-based neural orthoses for the correction of drop foot. IEEE Trans Neural Syst Rehabil Eng 2002;10:260-79.

[13] Kesar TM, Reisman DS, Perumal R, Jancosko AM, Higginson JS, Rudolph KS, et al. Combined effects of fast treadmill walking and functional electrical stimulation on post-stroke gait. Gait Posture 2011;33:309-13.

[14] Daly JJ, Ruff RL. Feasibility of combining multichannel functional neuromuscular stimulation with weight-supported treadmill training. J Neurol Sci 2004;225:105-15.

[15] Sabut SK, Sikdar CC, Kumar R, Mahadevappa M. Clinical use of Functional Electrical Stimulation of Foot Drop: A comparison between subacute and chronic stroke patients. J Mech Med Biol 2011;11:1165-77.

[16] McRae CGA, Johnston TE, Lauer RT, Tokay AM, Lee SCK, Hunt KJ. Cycling for children with neuromuscular impairments using electrical stimulation-Development of tricycle-based systems. Med Eng Phys 2009;31:650-9.

[17] Roche A, Laighin G ó, Coote S. Surface-applied functional electrical stimulation for orthotic and therapeutic treatment of drop-foot after stroke - a systematic review. Phys Ther Rev 2009;14:6380 .

[18] Jailani R, Tokhi MO, Gharooni SC, Jogtaei M. Finite State Control of FES-Assisted Walking with Spring Brake Orthosis. 2011 UkSim 13th Int Conf Comput Model Simul 2011:183-8.

[19] Greene PJ, Granat MH. A knee and ankle flexing hybrid orthosis for paraplegic ambulation. Med Eng Phys 2003;25:539-45.

[20] Gharooni S, Heller B, Tokhi M. A new hybrid spring brake orthosis for controlling hip and knee flexion in the swing phase. IEEE Trans Neural Syst Rehabil Eng 2001;9:106-7.

[21] Moore J, Zouridakis G. Biomedical Technology and Devices Handbook. Florida (USA): CRC Press LLC; 2004.
[22] Robertson V, Ward A, Low J, Reed A

Electrotherapy Explained: Principles and Practice. Fourth Edi. Heidi Harrison; 2006.

[23] Stein RB, Rolf R, Everaert DG, Bobet J, Chong S. Surface electrical stimulation for foot drop: Control aspects and walking performance. $\mathbf{J}$ Autom Control 2008;18:47-52.

[24] Taylor PN, Wright P, Burridge JH, Man GE, Swain ID. Correction of bi-lateral dropped foot using the Odstock 2 channel stimulator (O2CHS) Proc 5th Ann IFESS Conf 1999:8-11.

[25] Blaya J a, Herr H. Adaptive control of a variableimpedance ankle-foot orthosis to assist drop-foot gait. IEEE Trans Neural Syst Rehabil Eng 2004;12:24-31.

[26] Malezic M, Bogataj U, Gros N, Decman I, Vrtacnik P, Kljajic M, et al. Application of a programmable dual-channel adaptive electrical stimulation system for the control and analysis of gait. J Rehabil Res Dev 1992;29:41.

[27] Chou CH, Chen S, Hwang YS, Ho CS, Chen CC, Chen SC, et al. Application of FES for Hemiplegia in Extremity Coordination Training. 2011 5th Int. Conf. Bioinforma. Biomed. Eng., IEEE; 2011, p. 1-4.

[28] Kim J-H, Chung Y, Kim Y, Hwang S. Functiona electrical stimulation applied to gluteus medius and tibialis anterior corresponding gait cycle for stroke. Gait Posture 2012.

[29] Sabut SK, M. M, Mahadevappa M Neuroprosthesis-Functional Electrical Stimulation: Opportunities in Clinical Application for Correction of Drop-Foot. 2008 First Int. Conf. Emerg. Trends Eng. Technol., IEEE; 2008, p. 950-3.

[30] O’Halloran T, Haugland M, Lyons GM, Sinkjær T. Modified implanted drop foot stimulator system with graphical user interface for customised stimulation pulse-width profiles. Med Biol ... 2003;41:701-9.

[31] O'Halloran T, Haugland M, Lyons GM, Sinkjær T, Sinkjaer T. An Investigation of the Effect of Modifying Stimulation Profile Shape on the Loading Response Phase of Gait, during FESCorrected Drop Foot: Stimulation Profile and Loading Response. Neuromodulation 2004;7:113-25.

[32] Breen PP, O’Keeffe DT, Conway R, Lyons GM. A system for the delivery of programmable, adaptive stimulation intensity envelopes for drop foot correction applications. Med Eng Phys 2006;28:177-86.

[33] Lyons G, Wilcox D, Lyons D, Hilton D. Evaluation of a drop foot stimulator FES intensity envelope matched to tibialis anterior muscle activity during walking. 5th Annu IFESS Conf 2000:1-4. 

development of a potential optimized stimulation intensity envelope for drop foot applications. IEEE Trans Neural Syst Rehabil Eng 2003;11:249-56.

[35] Weber DJ, Stein RB, Chan KM, Loeb GE, Richmond FJR, Rolf R, et al. BIONic WalkAide for correcting foot drop. Annu Int Conf IEEE Eng Med Biol Soc 2004;6:4189-92.

[36] Loeb GE, Richmond FJR, Baker LL. The BION devices: injectable interfaces with peripheral nerves and muscles. Neurosurg Focus 2006;20:E2.

[37] Loeb GE, Peck RA, Moore WH, Hood K. BION system for distributed neural prosthetic interfaces. Med Eng Phys 2001;23:9-18.

[38] Miura N, Watanabe T, Akasaka K, Suzuki T. A clinical trial of a prototype of wireless surface fes rehabilitation system in foot drop correction. Conf Proc IEEE Eng Med Biol Soc 2011;2011:5461-4.

[39] Breen PP, Corley GJ, O'Keeffe DT, Conway R Olaighin G. A programmable and portable NMES device for drop foot correction and blood flow assist applications. Med Eng Phys 2009;31:4008.

[40] Dosen S, Popovic DB. Rule-based control of walking by using decision trees and practical sensors. 2008 9th Symp. Neural Netw. Appl. Electr. Eng., IEEE; 2008, p. 125-8.

[41] Haugland M, Sinkjaer T. Cutaneous whole nerve recordings used for correction of footdrop in hemiplegic man. IEEE Trans Rehabil Eng 1995;3:307-17.

[42] Haugland M. A miniature implantable nerve stimulator. 2nd Annu. Conf. Int. Funct. Electr. Stimul. Soc., 1997, p. 3-4.

[43] Hansen M, Haugland M. Machine learning for real time control of foot-drop correction using natural sensors. 5th Annu. IFESS Conf., 2000, p. $2-5$

[44] Hoffer J, Baru M, Bedard S, Calderon E, Desmoulin G, Dhawan P, et al. Initial results with fully implanted Neurostep TM FES system for foot drop. 10th Annu. Conf. Int. FES Soc., 2005, p. 5-7.

[45] Granat MH, Maxwell DJ, Ferguson ACB, Lees KR, Barbenet JC. Peroneal stimulator: Evaluation for the correction of spastic drop foot in hemiplegia. Arch Phys Med Rehabil 1996;77:19_ 24.

[46] Taylor PN, Burridge JH, Dunkerley AL, Wood DE, Norton JA, Singleton C, et al. Clinical use of the Odstock dropped foot stimulator: its effect on the speed and effort of walking. Arch Phys Med Rehabil 1999;80:1577-83.
[47] Taylor PN, Burridge J, Dunkerley A, Wood D, Norton J, Singleton C, et al. Clinical audit of 5 years provision of the Odstock dropped foot stimulator. Artif Organs 1999;23:440-2.

[48] Taylor PN, Burridge JH, Dunkerley a L, Lamb a, Wood DE, Norton J a, et al. Patients' perceptions of the Odstock Dropped Foot Stimulator (ODFS) Clin Rehabil 1999;13:439-46.

[49] Van Swigchem R, Vloothuis J, Den Boer J, Weerdesteyn V, Geurts ACH. Is transcutaneous peroneal stimulation beneficial to patients with chronic stroke using an ankle-foot orthosis? A within-subjects study of patients' satisfaction, walking speed and physical activity level. J Rehabil Med Off J UEMS Eur Board Phys Rehabil Med 2010;42:117-21.

[50] Robertson J, Whittaker M. Clinical perspectives in fitting the WalkAide2 foot-drop stimulator. 10th Annu. Conf. Int. FES Soc., 2005, p. 6-8.

[51] Kottink AI, Hermens HJ, Nene A V, Tenniglo MJ, van der Aa HE, Buschman HP, et al. A randomized controlled trial of an implantable 2channel peroneal nerve stimulator on walking speed and activity in poststroke hemiplegia. Arch Phys Med Rehabil 2007;88:971-8.

[52] Burridge J, Haugland M, Larsen B, Svaneborg N, Iversen H, P BC, et al. Long-term follow-up of patients using the ActiGait implanted drop-foot stimulator. 10th Annu. Conf. Int. FES Soc., 2005, p. $2-4$.

[53] Van Swigchem R, Weerdesteyn V, van Duijnhoven HJR, den Boer J, Beems T, Geurts AC. Near-normal gait pattern with peroneal electrical stimulation as a neuroprosthesis in the chronic phase of stroke: a case report. Arch Phys Med Rehabil 2011;92:320-4.

[54] Stanic U, Trnkoczy A. Closed-loop positioning of hemiplegic patient's joint by means of functional electrical stimulation. IEEE Trans Biomed Eng 1974;21:365-70.

[55] Watelain E, Barbier F, Allard P, Thevenon a Angué JC. Gait pattern classification of healthy elderly men based on biomechanical data. Arch Phys Med Rehabil 2000;81:579-86.

[56] Kobravi H-R, Erfanian A. Decentralized adaptive robust control based on sliding mode and nonlinear compensator for the control of ankle movement using functional electrical stimulation of agonist-antagonist muscles. J Neural Eng 2009;6.

[57] Nekoukar V, Erfanian A. Adaptive terminal sliding mode control of ankle movement using functional electrical stimulation of agonistantagonist muscles. 32nd Annu. Int. Conf. IEEE EMBS, vol. 2010, 2010, p. 5448-51.

[58] Nahrstaedt H, Schauer T, Shalaby R, Hesse S, Raisch J. Automatic control of a drop-foot stimulator based on angle measurement using bioimpedance. Artif Organs 2008;32:649-54. 
[59] Song CG, Kim SC, Nam KC, Kim DW. Optimum electrode configuration for detection of leg movement using bio-impedance. Physiol Meas 2005;26:S59-68.

[60] Chang M-H, Chen Y-LY-LY-L, Wang K-C, Kuo T-S. Implementation of fuzzy control over FESassisted locomotion for CVA patients. Proc. 20th Annu. Int. Conf. IEEE Eng. Med. Biol. Soc. Vol.20 Biomed. Eng. Towar. Year 2000 Beyond (Cat. No.98CH36286), vol. 5, IEEE; 1998, p. 2721-3.

[61] Chen Y-L, Chen W-L. Alternative control in FES-assisted locomotion. IEEE EMBS AsianPacific Conf. Biomed. Eng., IEEE; 2003, p. 250

[62] Chen Y-L, Chen S-C, Chen W-L, Hsiao C-C, Kuo T-S, Lai J-S. Neural network and fuzzy control in FES-assisted locomotion for the hemiplegic. J Med Eng Technol 2004;28:32-8.

[63] Chen Y-L, Chen W, Hsiao C-C, Kuo T-S, Lai JS. Development of the FES System with Neural Network+PID Controller for the Stroke. 2005 IEEE Int. Symp. Circuits Syst., IEEE; 2005, p. 5119-21.

[64] Melo PL, Silva MT, Martins JM, Newman DJ. Identification of Muscle Dynamics For Functional Electrical Stimulation Control Applications. J Biomech 2012;45:S72.

[65] Chen Y-L, Li YC, Kuo T-SS, Lai J-SS. The development of a closed-loop controlled functional electrical stimulation (FES) in gait training. J Med Eng Technol 2001;25:41-8.

[66] Chen Y-L, Chang W-H, Chen S-C, Sheu P-F, Chen W-L. The development of a knee locker with closed-loop functional electrical stimulation (FES) for hemiplegia in gait training. Disabil Rehabil 2003;25:916-21.

[67] Mourselas N, Granat MHM. Correction of drop foot using a fuzzy logic controlled miniature stimulator. Proc. 5th Ann. IFESS Conf, 2000, p

[68] Benedict GA, Ruiz VF. A portable gait analysis and correction system using a simple event detection method. IEEE Int. Conf. Syst. Man Cybern., vol. vol.4, IEEE; 2002, p. 6.

[69] Yeom H, Chang Y-H. Autogenic EMG-controlled functional electrical stimulation for ankle dorsiflexion control. J Neurosci Methods 2010;193:118-25.

[70] Muraoka Y. Development of 2-channel gait assist electrical stimulator for hemiplegia. Proc. 41st SICE Annu. Conf. SICE 2002., vol. 3, Soc. Instrument \& Control Eng. (SICE); 2002, p. 2028-31.

[71] Chen WL, Chen SC, Chen CC, Chou CH, Shih YY, Chen Y-LL, et al. Patient-driven loop control for ambulation function restoration in a non- invasive functional electrical stimulation system. Disabil Rehabil 2010;32:65-71.

[72] Burridge JH, Taylor PN, Hagan S, Swain ID Experience of clinical use of the Odstock dropped foot stimulator. Artif Organs 1997;21:254-60.

[73] Hausdorff JM, Ring H. Effects of a new radio frequency-controlled neuroprosthesis on gait symmetry and rhythmicity in patients with chronic hemiparesis. Am J Phys Med Rehabil 2008;87:4-13.

[74] Stanic U, Acimović-Janezic R, Gros N, Trnkoczy A, Bajd T, Kljajić M. Multichannel electrical stimulation for correction of hemiplegic gait. Methodology and preliminary results. Scand J Rehabil Med 1978;10:75-92.

[75] OttoBock. ActiGait System 2012.

[76] Bulley C, Shiels J, Wilkie K, Salisbury L. User experiences, preferences and choices relating to functional electrical stimulation and ankle foot orthoses for foot-drop after stroke. Physiotherapy 2011;97:226-33.

[77] Kluding PM, Dunning K, O’Dell MW, Wu SS, Ginosian J, Feld J, et al. Foot drop stimulation versus ankle foot orthosis after stroke: 30 -week outcomes. Stroke 2013;44:1660-9.

[78] Ring H, Treger I, Gruendlinger L, Hausdorff JM. Neuroprosthesis for footdrop compared with an ankle-foot orthosis: effects on postural control during walking. J Stroke Cerebrovasc Dis 2009;18:41-7.

[79] Jones DA. High-and low-frequency fatigue revisited. Acta Physiol Scand 1996;156:265-70.

[80] Bobet J. Can muscle models improve FESassisted walking after spinal cord injury? J Electromyogr Kinesiol 1998;8:125-32.

[81] Kesar TM, Perumal R, Jancosko A, Reisman DS Rudolph KS, Higginson JS, et al. Novel patterns of functional electrical stimulation have an immediate effect on dorsiflexor muscle function during gait for people poststroke. Phys Ther 2010;90:55-66.

[82] D’Orey S. Passive Dynamic Walkers and Sensory Systems for Gait Analysis. 2012.

[83] Hogan N. Adaptive control of mechanical impedance by coactivation of antagonist muscles. IEEE Trans Automat Contr 1984;29:681-90.

[84] Vasconcelos CF, Martins JM, Silva MT. Mechanical Design and Control of an Active Ankle-Foot Orthosis. J Biomech 2012;45:S523.

[85] Zajac FE. Muscle and Tendon: Properties, Models, Scaling, and Application to Biomechanics and Motor Control. Crit Rev Biomed Eng 1989;17:359. 

powered ankle-foot prosthesis based on a neuromuscular model. IEEE Trans Neural Syst Rehabil Eng 2010;18:164-73.

[87] Ferrarin M, Palazzo F, Riener R, Quintern J. Model-based control of FES-induced single joint movements. IEEE Trans Neural Syst Rehabil Eng 2001;9:245-57.

[88] Previdi F. Identification of black-box nonlinear models for lower limb movement control using functional electrical stimulation. Control Eng Pract 2002;10:91-9.

[89] Le F, Markovsky I, Freeman CT, Rogers E. Identification of electrically stimulated muscle models of stroke patients. Control Eng Pract 2010;18:396-407.

[90] Le F, Markovsky I, Freeman CT, Rogers E. Recursive identification of Hammerstein systems with application to electrically stimulated muscle. Control Eng Pract 2012;20:386-96.

[91] Haugland M, Childs C. An implantable foot drop stimulator. 5th Annu. IFESS Conf., 2000, p. 1-4.

[92] Sabut SK, Kumar R, Mahadevappa M. Design of a programmable multi-pattern FES system for restoring foot drop in stroke rehabilitation. J Med Eng Technol 2010;34:217-23.

[93] Burridge JH, Haugland M, Larsen B, Pickering RM, Svaneborg N, Iversen HK, et al. Phase II trial to evaluate the ActiGait implanted drop-foot stimulator in established hemiplegia. J Rehabil Med 2007;39:212-8.

[94] Hart DJ, Taylor PN, Chappell PH, Wood DE. A microcontroller system for investigating the catch effect: functional electrical stimulation of the common peroneal nerve. Med Eng Phys 2006;28:438-48.

[95] Khattar B, Banerjee A, Reddi R, Dutta A. Feasibility of Functional Electrical StimulationAssisted Neurorehabilitation following Stroke in India: A Case Series. Case Rep Neurol Med 2012;2012:830873

[96] Chou CH, Chen SC, Hwang YS, Ho CS, Chen CC, Chen Y-LL, et al. Application of FES for Hemiplegia in Extremity Coordination Training. 2011 5th Int. Conf. Bioinforma. Biomed. Eng., IEEE; 2011, p. 1-4.
[97] Sabut SK, Sikdar C, Mondal R, Kumar R, Mahadevappa M. Restoration of gait and motor recovery by functional electrical stimulation therapy in persons with stroke. Disabil Rehabil 2010;32:1594-603.

[98] Popovic DB, Sinkjær T. Improved Control for Functional Electrical Stimulation to Restore Walking. Hong Kong Physiother J 2000;18:1220.

[99] Cikajlo I, Bajd T. FES Gait Re-education: The Swing Phase Estimation. Neuromodulation 2003;6:122-7

[100] Taylor PN, Burridge JH, Dunkerley AL, Wood DE, Norton JA, Singleton C, et al. Clinical use of the Odstock dropped foot stimulator: its effect on the speed and effort of walking. Arch Phys Med Rehabil 1999;80:1577-83.

[101] Kostov A, Sinkjaer T, Upshaw B. Gait event discrimination using ALNs for control of FES in foot-drop problem. Proc. 18th Annu. Int. Conf. IEEE Eng. Med. Biol. Soc., vol. 1, IEEE; 1996, p 459-60.

[102] Hansen M, Haugland M, Sinkjaer T, Donaldson N. Real Time Foot Drop Correction using Machine Learning and Natural Sensors. Neuromodulation 2002;5:41-53.

[103] Benedict G, Ruiz V. A portable gait analysis and correction system using a simple event detection method. IEEE Trans Syst Man Cybern 2002:0-5.

[104] Sabut SK, Kumar R, Mahadevappa M. Design of an insole embedded foot pressure sensor controlled FES system for foot drop in stroke patients. Int. Conf. Syst. Med. Biol., IEEE; 2010 , p. $237-41$

[105] Chen M, Wu B, Lou X, Zhao T, Li J, Xu Z, et al A self-adaptive foot-drop corrector using functional electrical stimulation (FES) modulated by tibialis anterior electromyography (EMG) dataset. Med Eng Phys 2013;35:195-204.

[106] Loeb GE, Richmond FJR, Singh J, Peck R a, Tan W, Zou Q, et al. RF-powered BIONs for stimulation and sensing. Conf Proc IEEE Eng Med Biol Soc 2004;6:4182-5. 
